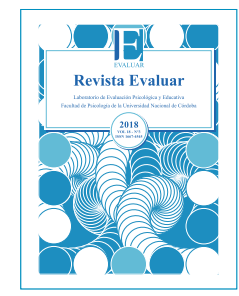

\title{
Validación de la Escala de Búsqueda de Sensaciones Sexuales en mexicanos casados o cohabitantes
}

\section{Validation of the Sexual Sensation Seeking Scale among married or cohabiting Mexicans}

\author{
José Moral de la Rubia * 1 \\ 1 - Facultad de Psicología, Universidad Autónoma de Nuevo León.
}

Introducción

Método

Resultados

Discusión

Referencias

Recibido: 28/06/2018 Revisado: 19/09/2018 Aceptado: 05/10/2018

Resumen

El objetivo del estudio fue validar la Escala de Búsqueda de Sensaciones Sexuales de 9 ítems (SSSS-9) en una población de adultos mexicanos casados o cohabitantes con pareja del sexo opuesto. Se recolectó una muestra de 807 participantes (53.4\% mujeres y $46.6 \%$ hombres; $90.1 \%$ casados y $9.9 \%$ cohabitantes) por rutas aleatorias. Se aplicó la SSSS-9 y las escalas de conducta infiel y actitud hacia la exclusividad marital. La SSSS-9 presentó una consistencia interna muy alta. El número de factores fue uno según el análisis paralelo de Horn. El modelo unifactorial tuvo buen ajuste y resultó válido para ambos sexos por análisis factorial confirmatorio. La distribución de la SSSS-9 mostró asimetría positiva. Su promedio fue mayor en hombres que en mujeres. Tuvo correlación alta con conducta infiel y moderada con actitud hacia la exclusividad marital. Se concluye que las propiedades de SSSS-9 resultan mejores en esta población que las reportadas con SSSS-11 en otras poblaciones.

Palabras clave: búsqueda de sensaciones sexuales, parejas concurrentes, actitud hacia la infidelidad marital, sexo, México

\begin{abstract}
The objective of this study was to validate the 9-item Sexual Sensation Seeking Scale (SSSS-9) in a population of Mexican adults married or cohabiting with a partner of the opposite sex. A sample of 807 participants was collected (53.4\% women and $46.6 \%$ men, $90.1 \%$ married and $9.9 \%$ cohabitants) from random sources. The SSSS-9, one scale of unfaithful behavior and other one of attitude toward marital exclusivity were applied. The SSSS-9 showed very high internal consistency. The number of factors was one through Horn's parallel analysis. The one-factor model had a good fit and was valid across both sexes through confirmatory factor analysis. The distribution of SSSS-9 showed positive asymmetry. Its average was higher among men than among women. SSSS-9 had a high correlation with unfaithful behavior, and moderate with attitude toward marital exclusivity. It is concluded that the properties of SSSS-9 are better in this population than those reported with SSSS-11 in other populations.
\end{abstract}

Key words: sexual sensation seeking, concurrent partners, attitude toward marital infidelity, sex, Mexico

* Correspondencia a: José Moral de la Rubia. c/Dr. Carlos Canseco 110. Col. Mitras Centro. C.P. 64460. Monterrey, Nuevo León, México. Tel. 005281 83338233. Ext. 423. Fax. Ext. 103. Correo electrónico: jose_moral@hotmail.com 


\section{Introducción}

Cuando una persona cree que su pareja le es infiel se siente traicionada y menos comprometida y usualmente la confirmación de la infidelidad conduce a la ruptura o alejamiento (Huang, Cassels, \& Winer, 2015). Las causas de la búsqueda de parejas concurrentes son diversas, entre ellas se considera a la búsqueda de sensaciones sexuales como uno de sus factores de riesgo (Kogan, Cho, Barnum, \& Brown, 2015). Más allá de la aplicación de este concepto a las conductas sexuales de riesgo (Xu, Zheng, Liu, \& Zheng, 2016), la búsqueda de sensaciones sexuales puede ser una variable importante en el estudio de las parejas. Consecuentemente, poseer un instrumento adecuado para su evaluación adquiere relevancia.

La búsqueda de sensaciones sexuales fue definida por Kalichman et al. (1994) como la tendencia a obtener niveles más altos de excitación sexual mediante la implicación en experiencias sexuales novedosas. Para su evaluación, estos autores crearon la Escala de Búsqueda de Sensaciones Sexuales de nueve ítems (SSSS-9). En una muestra de 106 hombres homosexuales, encontraron un nivel aceptable de consistencia interna ( $\alpha$ de Cronbach $=.75$ ) y de estabilidad temporal en un intervalo de dos semanas $\left(r_{[36]}=.78, p<\right.$ $.01)$. Las correlaciones de los ítems con la escala variaron de .29 a .58 con una media de .43 . Al calcular una función discriminante para diferenciar entre los participantes que no habían tenido sexo anal sin uso del condón en los últimos tres meses y los que sí, la SSSS-9 tuvo el coeficiente estructural más alto $(\beta=.56)$.

Posteriormente, Kalichman y Rompa (1995) modificaron la escala para mejorar su consistencia interna. Se conservaron siete de sus nueve ítems (ítems 1, 4, 5, 6, 7, 8 y 9), se eliminaron dos (ítems 2 y 3 ) y se introdujeron cuatro nuevos: las sensaciones físicas son lo más importante en el sexo; disfruto la sensación de mantener relaciones sexuales sin preservativo; mis parejas sexuales probablemente piensan que soy una persona que hace cosas arriesgadas; y cuando se trata de sexo, la atracción física es más importante que lo bien que yo conozca a la persona. De este modo la escala pasó a tener 11 ítems (SSSS-11). Esta nueva versión fue aplicada en dos muestras: una de 296 hombres que tienen sexo con hombres y otra de 60 hombres y 98 mujeres heterosexuales. En la primera muestra se encontró una consistencia interna aceptable ( $\alpha$ de Cronbach $=.79$ ), una estabilidad temporal a los tres meses también aceptable $\left(r_{[195]}=.69, p<.01\right)$ y correlaciones bajas con conductas sexuales de riesgo $\left(r_{[296]}=.22\right.$ con número de parejas y .19 con coito sin condón). En la segunda, la consistencia interna fue alta ( $\alpha$ de Cronbach $=.81)$, al igual que la estabilidad temporal $\left(r_{[52]}=.73, p<.01\right)$. Conforme con la expectativa de mayor libertad e impulso sexual en los hombres esgrimida por los autores, su media $(\mathrm{M}=2.34, \mathrm{DE}=0.67)$ fue significativamente más alta $\left(t_{[143]}=3.77, p<.001\right)$ que la de las mujeres $(\mathrm{M}=1.95, \mathrm{DE}=0.57)$. Finalmente, al comprobar la validez de constructo, se encontró que las correlaciones con conductas sexuales de riesgo fueron medianas.

Gaither y Sellbom (2003) elaboraron una versión de 10 ítems para su aplicación a estudiantes universitarios heterosexuales de ambos sexos (SSSS-10). En una muestra de 528 estudiantes universitarios estadounidenses, la consistencia interna de la escala fue alta en hombres ( $\alpha$ de Cronbach $=.83)$ y mujeres $(\alpha$ de Cronbach $=.81)$, fue discriminante entre ambos sexos y correlacionó con permisividad sexual, siendo las correlaciones más altas en mujeres que en hombres (Gaither \& Sellbom, 2003).

Kalichman et al. (1994) y Kalichman y Rompa (1995) asumieron que la escala presenta una estructura unidimensional. No obstante, es- 
tudios factoriales recientes con la SSSS-11 han revelado una estructura de dos factores correlacionados (nuevas experiencias y nuevas sensaciones) que, tras eliminarse dos o tres de los ítems nuevos, presenta mejor ajuste a los datos (Ballester-Arnal, Ruiz-Palomino, Espada-Sánchez, Morell-Mengual, \& Gil-Llario, 2018; De Oliveira-Santos, Soares-Ferreira, Carvalho-Duarte, \& Ferreira, 2017; Santos-Iglesias, Moyano, Castro, Granados, \& Sierra, 2018). La denominación dada a cada factor intenta reflejar el contenido dominante de sus ítems definidores. En el factor de nuevas sensaciones predominan los contenidos sobre sensaciones sin referencia explícita a lo externo, por ejemplo, estoy interesado en experimentar nuevas sensaciones sexuales o me gustan las experiencias y sensaciones sexuales nuevas y excitantes y en el factor de nuevas experiencias predominan los contenidos sobre situaciones con referencia explícita o dependientes de otras personas o estímulos externos, por ejemplo disfruto de la compañía de una persona sensual o disfruto ver películas pornográficas.

En México, no se ha validado la escala. No obstante, se tiene un estudio realizado en una muestra de 255 hombres que tienen sexo con hombres de Monterrey (Valdez, Moral, Onofre, Castillo, Benavides, \& López, 2016). En esta investigación, se reportó una consistencia interna aceptable ( $\alpha$ de Cronbach $=.74$ ) semejante a la obtenida con la SSSS-9 (Kalichman et al., 1994). La media en la puntuación total de la SSSS-11 $(\mathrm{M}=2.80$, IC $95 \%: 2.74,2.87)$ fue significativamente mayor que la obtenida entre 60 varones adultos estadounidenses usuarios de servicios socio-sanitarios $\left(\mathrm{M}=2.34\right.$, IC $95 \%: 2.17,2.51 ; t_{[240]}$ $=14.59, p<.001)$ del estudio de Kalichman $\mathrm{y}$ Rompa (1995). Esta diferencia se puede atribuir a las distintas poblaciones de las cuales fueron extraídas las muestras, al ser la población de hombres que tienen sexo con hombres la que presenta mayor riesgo de contraer enfermedades de transmisión sexual por sus prácticas sexuales (Iglesias \& Reyes, 2017).

Hay investigaciones sobre la confiabilidad y validez de la SSSS en sus tres versiones, aunque estas presentan debilidades. La mayoría de ellas se han hecho con muestras incidentales, usualmente con hombres que tienen sexo con hombres y estudiantes universitarios o de media superior. Otra debilidad aparece en relación con las técnicas de análisis. Los ítems de la SSSS presentan un rango muy limitado de respuesta (cuatro valores ordinales), lo que provoca que el coeficiente alfa de Cronbach y la correlación producto-momento de Pearson subestimen su confiabilidad. En su lugar, sería más adecuado usar el coeficiente alfa ordinal y la correlación poliserial y policórica (Gadermann, Guhn, \& Zumbo, 2014). Además, los estudios más recientes con la SSSS-11 sugieren reducir la escala a nueve u ocho ítems, eliminando dos o más de los ítems nuevos para definir un modelo bifactorial (Ballester-Arnal et al., 2018; De Oliveira-Santos et al., 2017; Santos-Iglesias et al., 2018), cuando se podría definir un modelo más parsimonioso de un factor con la escala original, en la que domina el contenido de situaciones interpersonales o dependientes de estímulos externos.

Este estudio tiene como propósito validar la SSSS-9 en población de personas mexicanas casadas o cohabitantes con pareja del sexo opuesto residentes en Monterrey. Con base en este propósito se definieron ocho objetivos: 1) comprobar la consistencia interna de los nueve ítems de la SSSS-9, así como su discriminabilidad y adecuación distribucional; 2) determinar el número de factores por criterios empíricos y en caso de dos o más factores explorar los mismos; 3 ) contrastar el modelo de un factor con nueve indicadores, el de dos factores propuesto para la SSSS-11 limitado a los nueve ítems de la SSSS-9 y posibles modelos 
alternativos derivados del análisis factorial exploratorio; 4) contrastar la invarianza de los modelos entre ambos sexos; 5) una vez establecida la estructura factorial, calcular la consistencia interna de la escala; 6) describir la distribución de la escala y comparar su media entre ambos sexos; 7) comprobar la validez de constructo concurrente en relación con conducta infiel y actitud hacia la exclusividad marital comparando estas correlaciones entre ambos sexos y 8) observar si la deseabilidad social tiene efecto sobre la SSSS-9 y comprobar la sustantividad de las correlaciones de validez al parcializar la deseabilidad social.

En correspondencia con los ocho objetivos enunciados se esperan: 1) ítems consistentes y $\sin$ efecto suelo o techo ( $\geq 80 \%$ de los casos en uno de los polos), como ya se observó en el estudio original de Kalichman et al. (1994) y estudios previos (De Oliveira-Santos et al., 2017; Teva \& Paz-Bermúdez, 2008), con la posibilidad de que los ítems de la SSSS-9 eliminados en la elaboración de la SSSS-11 (ítems 2 y 3) sean los menos consistentes; 2) un factor (Kalichman et al., 1994) o dos factores (nuevas sensaciones personales (ítems 1,8 y 9) y nuevas experiencias interpersonales o dependientes de estímulos externos (ítems 2, 3, 4, 5, 6 y 7), a semejanza de los obtenidos con la SSSS-11 (Ballester-Arnal et al., 2018; De Oliveira-Santos et al., 2017; Santos-Iglesias et al., 2018); 3) mejor ajuste del modelo de dos factores que el de un factor, como en los estudios con la SSSS-11, aunque con esta versión original de la escala, los factores pueden presentar falta de validez discriminante o exceso de varianza compartida, indicando unidimensionalidad al ser predominante el contenido de situación interpersonal o dependiente de estímulo externo; 4) adecuadas propiedades de invarianza de los modelos factoriales entre ambos sexos, al hipotetizarse que las diferencias entre hombres y mujeres serán de magnitud (promedios), pero no de cualidad (con- figuración y número de los factores subyacentes); 5) consistencia interna alta en la escala al ser estimada por un método más adecuado a la naturaleza ordinal de sus ítems (Gadermann et al., 2014); 6) asimetría positiva en la distribución (Teva \& Paz-Bermúdez, 2008) por los casos de conducta sexual compulsiva en la cola derecha de la distribución y mayor promedio en los hombres que en las mujeres por la mayor impulsividad y búsqueda de sensaciones asociadas a estos primeros (Cross, Cyrenne, \& Brown, 2013) y la mayor permisividad sexual que la cultura occidental concede al hombre (Ballester-Arnal et al., 2018; De Oliveira-Santos et al., 2017; Kalichman \& Rompa, 1995; Santos-Iglesias et al., 2018); 7) correlación positiva de la puntuación total de la SSSS-9 con infidelidad marital y actitud hacia la infidelidad marital (Kogan et al., 2015), pudiendo ser las correlaciones más altas en hombres que en mujeres por una mayor permisividad cultural hacia los hombres en asuntos de infidelidad (Selterman \& Koleva, 2015) y 8) fuerza de asociación trivial o pequeña entre la SSSS-9 y la deseabilidad social, lo que hace que las correlaciones de validez concurrente resulten sustantivas al parcializar la deseabilidad social, conforme con los resultados de otros estudios con escalas de conductas sexuales (Grubbs, Exline, Pargament, Hook, \& Carlisle, 2015; Muise, Stanton, Kim, \& Impett, 2016).

\section{Método}

Se realizó un estudio instrumental con un diseño ex post facto transversal y un muestreo por rutas al azar.

\section{Participantes}

Los criterios de inclusión fueron: tener al 
Distribución de frecuencias de las variables sociodemográficas.

\begin{tabular}{|c|c|c|c|}
\hline Variable & Valor & $\mathbf{n}(p)$ & $\mathbf{N}(P)$ \\
\hline \multirow{2}{*}{ Sexo } & Mujer & $431(53.4 \%)$ & \\
\hline & Hombre & $376(46.6 \%)$ & \\
\hline \multirow{5}{*}{ Años de edad } & {$[18,30)$} & $285(35.3 \%)$ & $285(35.3 \%)$ \\
\hline & {$[30,40)$} & $230(28.5 \%)$ & $515(63.8 \%)$ \\
\hline & {$[40,50)$} & $220(27.3 \%)$ & $735(91.1 \%)$ \\
\hline & {$[50,60)$} & $60(7.4 \%)$ & $795(98.5 \%)$ \\
\hline & {$[60,94]$} & $12(1.5 \%)$ & $807(100 \%)$ \\
\hline \multirow{5}{*}{ Escolaridad } & Primaria & $173(21.4 \%)$ & $173(21.4 \%)$ \\
\hline & Secundaria & $231(28.6 \%)$ & $404(50.1 \%)$ \\
\hline & Media superior & $218(27 \%)$ & $622(77.1 \%)$ \\
\hline & Licenciatura & $164(20.3 \%)$ & $786(97.4 \%)$ \\
\hline & Posgrado & $21(2.6 \%)$ & $807(100 \%)$ \\
\hline \multirow{2}{*}{ Estado civil } & Casado & $727(90.1 \%)$ & \\
\hline & Cohabitante & $80(9.9 \%)$ & \\
\hline \multirow{6}{*}{$\begin{array}{l}\text { Años de matrimonio o } \\
\text { cohabitación }\end{array}$} & {$[0,7.17)$} & $361(44.7 \%)$ & $361(44.7 \%)$ \\
\hline & {$[7.17,14.34)$} & $146(18.1 \%)$ & $507(62.8 \%)$ \\
\hline & {$[14.34,21.51)$} & $171(21.2 \%)$ & $678(84 \%)$ \\
\hline & {$[21.51,28.67)$} & $91(11.3 \%)$ & $769(95.3 \%)$ \\
\hline & {$[28.67,35.84)$} & $27(3.3 \%)$ & $796(98.6 \%)$ \\
\hline & {$[35.84,43]$} & $11(1.4 \%)$ & $807(100 \%)$ \\
\hline \multirow{7}{*}{$\begin{array}{l}\text { Ingresos económicos } \\
\text { familiares al mes en } \\
\text { el último año en pesos } \\
\text { mexicanos }\end{array}$} & $<3000$ & $61(7.6 \%)$ & $61(7.6 \%)$ \\
\hline & {$[3,000,6,000)$} & $220(27.3 \%)$ & $281(34.8 \%)$ \\
\hline & {$[6,000,12,000)$} & $226(28.0 \%)$ & $507(62.8 \%)$ \\
\hline & {$[12,000,24,000)$} & $174(21.6 \%)$ & $681(84.4 \%)$ \\
\hline & {$[24,000,48,000)$} & $77(9.5 \%)$ & $758(93.9 \%)$ \\
\hline & {$[48,000,92,000)$} & $34(4.2 \%)$ & $792(98.1 \%)$ \\
\hline & $\geq 92,000$ & $15(1.9 \%)$ & $807(100 \%)$ \\
\hline \multirow{6}{*}{ Número total de hijos } & 0 & $130(16.1 \%)$ & $130(16.1 \%)$ \\
\hline & 1 & $149(18.5 \%)$ & $279(34.6 \%)$ \\
\hline & 2 & $237(29.4 \%)$ & $516(63.9 \%)$ \\
\hline & 3 & $211(26.1 \%)$ & $727(90.1 \%)$ \\
\hline & 4 & $60(7.4 \%)$ & $787(97.5 \%)$ \\
\hline & $\geq 5$ & $20(2.5 \%)$ & $807(100 \%)$ \\
\hline \multirow{4}{*}{ Afiliación religiosa } & Cristiana católica & $662(82.0 \%)$ & \\
\hline & Cristiana no católica & $76(9.4 \%)$ & \\
\hline & Otra & $13(1.6 \%)$ & \\
\hline & Ninguna & $56(6.9 \%)$ & \\
\hline
\end{tabular}


menos 18 años, estar casado o cohabitar con una pareja del sexo opuesto, ser mexicano, residir en la ciudad de Monterrey en México, saber leer y escribir y dar el consentimiento informado. Los criterios de eliminación fueron: cuestionario incompleto e informar que la pareja estuvo presente en el momento de ser contestado, leyendo o preguntando por las respuestas.

Se completó una muestra de 807 participantes, de los cuales 431 (53.4\%) fueron mujeres y $376(46.6 \%)$ hombres, sin diferencia de frecuencia estadísticamente significativa entre ambos sexos por la prueba binomial $(p=.057)$. Sus características sociodemográficas se presentan en la Tabla 1.

En la población de Monterrey, descontando al $31 \%$ de la población menor de 18 años, hay 98 hombres por cada 100 mujeres, una media de 35.1 años de edad, una media de 9.5 años de escolaridad (entre primer semestre y segundo de educación media superior), una media de ingresos mensuales de 6,700 pesos mexicanos (unos 335 dólares estadounidenses), una media de 2 hijos y el $83 \%$ de la población con afiliación religiosa cristiana católica, $7.4 \%$ cristiana no católica, 2.5 $\%$ de otra religión y $6.9 \%$ sin religión (Instituto Nacional de Estadística, Geografía e Informática [INEGI], 2016). Estos datos poblacionales son estadísticamente equivalentes a los de la muestra, como en la frecuencia de ambos sexos $\left(\chi^{2}\left[_{1, \mathrm{~N}=807]}\right.\right.$ $=1.87, p=.171)$ y de la afiliación religiosa $\left(\chi_{[3, \mathrm{~N}}^{2}\right.$ $\left.{ }_{807]}=7.13, p=.068\right)$, así como en la media de edad $\left(t_{[806]}=0.806, p=.421\right)$ y de número de hijos $\left(t_{[806]}\right.$ $=-0.27, p=.787)$, o muy semejantes, como en la media de escolaridad ( $\mathrm{Mdn}=4$ que corresponde a media superior, incluyendo estudios truncados $\mathrm{y}$ terminados) e ingresos ( $\mathrm{Mdn}=3$ que corresponde al intervalo $[6,000,11,999])$. Si se reduce la población a adultos de la zona metropolitana, se obtienen $89 \%$ casados y $11 \%$ cohabitantes (INEGI, 2016), lo que es un porcentaje equiva- lente al muestral $\left(\chi_{[1, \mathrm{~N}=807]}^{2}=0.97, p=.324\right)$. Por tanto, no se consideró necesario acudir a métodos de ponderación para corregir sesgos sociodemográficos

\section{Instrumentos}

Escala de búsqueda de sensaciones sexuales (SSSS-9) de Kalichman et al. (1994). Se compone de nueve ítems de codificación directa con cuatro categorías ordenadas de respuesta, de $1=$ Nada a 4 = Muchísimo. La puntuación total se obtiene sumando los nueve ítems y dividiendo por nueve, con lo que esta varía en un continuo de 1 a 4. Mayor puntuación refleja mayor tendencia a la búsqueda de excitación, experiencias y novedades sexuales. Su consistencia interna es aceptable ( $\alpha$ de Cronbach $=.75$ ) y se asume que a sus nueve ítems subyace un factor general (Kalichman et al., 1994). La versión en español que se aplicó en el presente estudio se obtuvo por traducción reversa (Streiner, Norman, \& Cairney, 2015; véase Anexo).

\section{Escala de conducta infiel de Romero-Palencia,} Rivera-Aragón y Díaz-Loving (2007). Cuenta con 48 ítems directos con cinco categorías de respuesta, de $1=$ Nunca a 5 = Siempre. A mayor puntuación, mayor frecuencia de deseos y situaciones con parejas concurrentes. Posee una consistencia interna muy alta ( $\alpha$ de Cronbach $=.98)$. Tiene una estructura de cuatro factores: infidelidad sexual con 21 ítems $(\alpha$ de Cronbach $=.97)$, deseo de infidelidad emocional con 14 ítems ( $\alpha$ de Cronbach $=.96)$, deseo de infidelidad sexual con ocho ítems ( $\alpha$ de Cronbach $=.96$ ), e infidelidad emocional con cinco ítems ( $\alpha$ de Cronbach $=.87$; (Romero et al., 2007). 
Escala de actitud hacia la exclusividad marital.

Fue creada por Weis y Felton (1987). Consta de siete ítems directos con cinco categorías ordenadas de respuesta que se puntúan de $1=$ Total rechazo a $5=$ Total aceptación. Su consistencia interna es alta $(\alpha=.87)$ y presenta una estructura unidimensional (Weis \& Felton, 1987). Las puntuaciones en la escala se obtienen por suma simple de los ítems. A mayor puntuación, mayor aceptación de la infidelidad a la pareja. La versión en español que se aplicó en el presente estudio se obtuvo por traducción reversa.

Inventario balanceado de deseabilidad social (BIDR) con la adaptación a México de Moral, García y Antona (2012). Se usó la versión de 20 ítems directos con siete categorías ordenadas de respuesta, de $1=$ Nada de acuerdo a $7=$ Totalmente de acuerdo. Esta versión quedó constituida por los 10 ítems directos de autoengaño ( $\alpha$ de Cronbach $=.76)$ y los 10 ítems directos de mane- jo de la impresión ( $\alpha$ de Cronbach $=.71)$, con los que se logra mejorar la consistencia interna total ( $\alpha$ de Cronbach $=.77$ ). El modelo de dos factores correlacionados en que se fundamenta la escala mostró buen ajuste a los datos por mínimos cuadrados generalizados $\left(\chi^{2} / g l=1.74\right.$, $\mathrm{RMSEA}=.03$, $\mathrm{GFI}=.95$ y AGFI $=.94)$. Las puntuaciones en la escala y los dos factores se obtienen por suma simple. A mayor puntuación, mayor tendencia a inflar el autoinforme en un sentido socialmente deseable (Moral et al., 2012).

En la presente muestra, los valores de consistencia interna por el coeficiente $\alpha$ ordinal y el coeficiente $\alpha$ de Cronbach variaron de muy altos a altos (Tabla 2).

\section{Procedimiento}

El muestreo de rutas empleado consistió en seleccionar aleatoriamente 81 calles a partir de la

Tabla 2

Consistencia interna en la muestra conjunta, de mujeres y de hombres.

\begin{tabular}{|c|c|c|c|c|c|c|}
\hline \multirow{3}{*}{$\begin{array}{c}\text { Escalas } \\
\text { y factores }\end{array}$} & \multicolumn{2}{|c|}{$\begin{array}{l}\text { Conjunta } \\
(\mathrm{N}=\mathbf{8 0 7})\end{array}$} & \multicolumn{2}{|c|}{$\begin{array}{l}\text { Mujeres } \\
(\mathrm{N}=\mathbf{4 3 1})\end{array}$} & \multicolumn{2}{|c|}{$\begin{array}{l}\text { Hombres } \\
(\mathrm{N}=376)\end{array}$} \\
\hline & & $\boldsymbol{\alpha}$ & $\alpha$ & $\alpha$ & $\boldsymbol{\alpha}$ & $\boldsymbol{\alpha}$ \\
\hline & ordinal & Cronbach & ordinal & Cronbach & ordinal & Cronbach \\
\hline \multicolumn{7}{|c|}{ Escala de búsqueda de sensaciones sexuales (SSSS-9) } \\
\hline Total & .994 & .972 & .994 & .972 & .993 & .969 \\
\hline Infidelidad sexual & .993 & .985 & .994 & .984 & .992 & .985 \\
\hline Deseo inf. emoc. & .985 & .974 & .985 & .971 & .984 & .975 \\
\hline Deseo inf. sexual & .989 & .978 & .991 & .977 & .987 & .977 \\
\hline Infidelidad emoc. & .972 & .941 & .972 & .935 & .961 & .942 \\
\hline \multicolumn{7}{|c|}{ Escala de actitud hacia la exclusividad marital } \\
\hline Total & .968 & .938 & .974 & .940 & .961 & .934 \\
\hline \multicolumn{7}{|c|}{ Inventario balanceado de deseabilidad social } \\
\hline Total & .883 & .865 & .888 & .869 & .879 & .861 \\
\hline Autoengaño & .849 & .823 & .852 & .828 & .847 & .819 \\
\hline M. de impresión & .853 & .827 & .853 & .825 & .856 & .832 \\
\hline
\end{tabular}


Guía Roji. De cada calle se recolectaron 10 unidades (con consentimiento informado y datos completos). Se pretendía que fueran cinco mujeres y cinco hombres independientes (uno por casa). $\mathrm{Si}$ la persona deseaba participar voluntariamente y satisfacía los criterios de inclusión, se dejaba un cuestionario para recogerlo media hora después o cuando lo indicase la persona.

Para determinar el tamaño de la muestra se usó la fórmula para la estimación bilateral de una media con población infinita, al ser el tamaño poblacional mayor que 100,000. Bajo un modelo de curva normal, si se desea estimar la media de la SSSS-9 con una desviación estándar esperada de 0.518 (Kalichman et al., 1994), un error absoluto de estimación de .036 y un intervalo de confianza del $95 \%$, se requeriría una muestra mínima de 796 participantes.

El estudio fue aprobado en sus aspectos éticos por el Comité de Doctorado, autoridad concerniente dentro de la institución académica, al ser sometido de forma extraordinaria al juicio de éste para cumplimentar requisitos del programa de financiamiento. Fue financiado por el Programa de Apoyo a la Investigación Científica y Tecnológica. Se solicitó el consentimiento expreso informado de los participantes en la primera hoja del cuestionario. En la hoja de consentimiento se garantizó el anonimato de las respuestas y se informó sobre la identidad del responsable del estudio, con quien se podía contactar por correo electrónico por cualquier cuestión suscitada por la investigación. No se solicitó ningún dato de identificación personal. Así, se respetaron las normas éticas de investigación de la Asociación Americana de Psicología (2017).

\section{Análisis de datos}

En relación con el primer objetivo, la con- sistencia interna de los ítems se estableció por la correlación poliserial (método de máxima verosimilitud de dos pasos) del ítem con el resto de la escala y la disminución del coeficiente alfa ordinal de la escala al ser calculado sin incluir al ítem. Si la correlación era menor que .30 y el valor del coeficiente alfa ordinal se incrementaba, se consideró que el ítem presentaba problemas de consistencia interna. La discriminabilidad se comprobó por la diferencia significativa de tendencia central entre el grupo de puntuaciones bajas (menores o iguales al cuartil inferior) y el de puntuaciones altas (mayores o iguales al cuartil superior) usando la prueba U de Mann-Whitney.

En relación con el segundo objetivo de determinar el número de factores, dicha determinación se hizo a través de la convergencia del análisis paralelo de Horn, coordenadas óptimas, factor de aceleración, promedio mínimo de las correlaciones parciales al cuadrado (criterio de Velicer) y a cuarta potencia (criterio de Velicer revisado) y autovalores de la matriz de correlaciones mayores que uno (criterio de Kaiser). Todos estos criterios se calcularon desde la matriz de correlaciones policóricas.

El método de análisis paralelo se implementó generando 1000 matrices de datos aleatorios a través de la permutación de los datos empíricos, por lo que se conservan los 807 casos y las nueve variables. A partir de las mismas se obtuvieron 1000 matrices de correlación y se calcularon los nueve autovalores de cada matriz de correlación. De este modo, se obtuvo una distribución para cada autovalor. Se calculó el percentil 95 de la distribución de cada autovalor. Los autovalores empíricos (de la matriz de correlación policórica original) que quedaron por encima de estos percentiles se consideran sustantivos y aquellos que quedaron por debajo se consideran espurios. El análisis de coordenadas óptimas compara las pendientes de los autovalores retenidos por el análisis 
paralelo de Horn. Requiere que estos gradientes o pendientes sean descendentes. En caso de que un gradiente sea ascendente, indica un cambio anómalo de curvatura y se retienen sólo los autovalores previos con gradientes descendentes. El factor de aceleración, desde los gradientes de cada autovalor, busca el cambio más brusco de pendiente y retiene los autovalores que quedan por encima de ese punto de cambio brusco de curvatura. Estos dos últimos métodos son variantes matematizadas del test de Cattell, el cual se basa en la búsqueda visual del punto de cambio de curvatura en la gráfica de sedimentación de los autovalores de la matriz de correlación de los datos empíricos. En la prueba de Velicer, los factores sustantivos se buscan parcializando los factores que fueron extraídos por análisis de componentes principales; se calculan sucesivas matrices de correlación parcial y el promedio de las correlaciones fuera de la diagonal principal elevadas al cuadrado o a la cuarta potencia. Un promedio mayor que el obtenido en el cálculo anterior indica que no queda varianza compartida y que se está extrayendo un factor espurio (Courtney, 2013).

En relación con los objetivos tercero $\mathrm{y}$ cuarto de comprobar el ajuste del modelo a los datos y su invarianza entre ambos sexos, ambos contrastes se hicieron por mínimos cuadrados no ponderados desde la matriz de correlaciones policóricas. Los errores estándar de las estimaciones se calcularon por el método percentiles corregidos de sesgo (con la extracción de 2,000 muestras aleatorias). Se estableció la validez convergente de cada factor (varianza de los indicadores explicada por su factor mayor que la explicada por otros factores no atribuibles o azar) por la varianza media extraída (AVE) y el coeficiente omega $(\omega)$ de McDonald. Valores de AVE > .50 y $\omega \geq .70$ muestran validez convergente. A su vez, se estableció la validez discriminante entre dos factores por una varianza compartida menor que dos ter- cios y menor que la AVE de cada factor. Se consideraron nueve índices de ajuste: $\chi^{2} / g l=$ chi-cuadrada relativa o cociente entre el valor mínimo de la función de discrepancia y los grados de libertad del modelo, GFI = índice de bondad de ajuste de Jöreskog y Sörbom, AGFI = índice de bondad de ajuste corregido de Jöreskog y Sörbom, CFI = índice comparativo de ajuste de Bentler, NFI = índice de ajuste normado de Bentler y Bonett, NNFI = índice de ajuste no normado de Bentler y Bonett, RFI = índice de ajuste relativo de Bollen, RMSEA = error de aproximación cuadrático medio de Steiger y Lindt y SRMR = error estandarizado cuadrático medio. A su vez, se estimó la parsimonia del modelo por la razón de parsimonia (PR) de James, Mulaik y Brett. Siguiendo a Byrne (2016), se estipularon como valores de buen ajuste: $\chi^{2} / g l \leq 2$, GFI, CFI, NFI, NNFI y RFI $\geq .95, \mathrm{AGFI} \geq .90, \mathrm{y}$ RMSEA y $\mathrm{SRMR} \leq .05 ; \mathrm{y}$ aceptable: $\chi^{2} / g l \leq 3$, GFI, CFI, NFI; NNFI y RFI $\geq .90, \mathrm{AGFI} \geq .85, \mathrm{RMSEA}<.08$ y $\mathrm{SRMR}<.10$. Una PR de .50 a .749 se interpretó como parsimonia alta y de .75 a 1 muy alta.

En relación con el quinto objetivo de calcular la consistencia interna de la escala y sus factores, esta se calculó a través del coeficiente alfa ordinal; valores mayores que .70 se consideraron aceptables y mayores que .80 altos.

En relación con el sexto objetivo de describir la distribución y su diferencia de tendencia central entre ambos sexos, el ajuste de la distribución a una curva normal se contrastó por la prueba de Kolmogorov-Smirnov-Lilliefors. Las medias se compararon por la prueba $t$ de Student usando el método repetitivo (con la extracción de 1000 muestras aleatorias) para estimar el error estándar y el intervalo de confianza de la diferencia media por el incumplimiento del supuesto de distribución normal. El tamaño del efecto del sexo sobre la escala se determinó por la $g$ de Hedges corregida de sesgo. Valores de $g$ menores que .20 
se interpretaron como un tamaño del efecto trivial, de .20 a .49 pequeño, de .50 a .79 mediano y mayor o igual que .80 grande (Téllez, García, \& Corral-Verdugo, 2015).

En relación con los objetivos séptimo y octavo de comprobar la validez de constructo concurrente y el sesgo por deseabilidad social, las correlaciones con las escalas de conducta infiel, actitud hacia la exclusividad marital y deseabilidad social se calcularon por la correlación producto-momento de Pearson. Debido al incumplimiento de normalidad se usó el método de muestreo repetitivo (con la extracción de 1000 muestras aleatorias) para estimar los errores estándar e intervalos de confianza. Se compararon las correlaciones entre ambos sexos por la prueba $Z$ de Fisher. También se usó el método de muestreo repetitivo (con la extracción de 1000 muestras) para estimar los errores estándar e intervalos de confianza de las diferencias medias.
Los contrastes fueron bilaterales con un nivel de significación de .05. Los cálculos se hicieron con SPSS22, módulo R4.2 para SPSS22, AMOS16 y Excel 2013.

\section{Resultados}

Distribución, discriminabilidad y consistencia interna de los ítems

En la muestra total, los nueve ítems fueron discriminantes. El efecto de este poder discriminativo fue grande ( $r^{2}$ de Rosenthal de .25 a .39 ) en siete ítems y mediano en los ítems $2\left(r^{2}=.17\right)$ y $6\left(r^{2}=.18\right)$. A su vez presentaron consistencia interna, como reflejo de las correlaciones de cada ítem con el resto de la escala que variaron de altas (CPSi,t-i $=.65)$ a muy altas (CPSi,t-i $=.79)$, con una media de .70 y un descenso en el valor de la consistencia interna de la escala al ser eliminado el ítem (Tabla 3).

Tabla 3

Distribución, discriminabilidad y consistencia interna de los ítems.

\begin{tabular}{|c|c|c|c|c|c|c|c|c|c|c|c|c|c|}
\hline \multirow{2}{*}{ Ítem } & \multicolumn{4}{|c|}{ Porcentaje } & \multirow[t]{2}{*}{$Q_{1}$} & \multirow[t]{2}{*}{$\boldsymbol{Q}_{2}$} & \multirow[t]{2}{*}{$Q_{3}$} & \multirow[t]{2}{*}{$R_{S I Q}$} & \multirow[t]{2}{*}{$A_{I Q}$} & \multirow{2}{*}{$\begin{array}{c}\text { Discr. } \\
Z_{U} \\
\end{array}$} & \multicolumn{3}{|c|}{ Consistencia } \\
\hline & 1 & 2 & 3 & 4 & & & & & & & $\mathrm{CPS}_{\mathrm{i}, \mathrm{t}}$ & $\mathrm{CPS}_{\mathrm{i}, \mathrm{t}-\mathrm{i}}$ & Ord. $\alpha_{t-\mathrm{i}}$ \\
\hline 1 & 38.0 & 34.6 & 16.5 & 10.9 & 1 & 2 & 3 & 1 & 0 & $-16.27^{* * *}$ & .75 & .66 & .925 \\
\hline 2 & 78.6 & 15.2 & 4.7 & 1.5 & 1 & 1 & 1 & 0 & I & $-11.71^{* * *}$ & .71 & .65 & .923 \\
\hline 3 & 67.2 & 18.6 & 7.2 & 7.1 & 1 & 1 & 2 & 0.5 & 1 & $-14.15^{* * *}$ & .74 & .66 & .923 \\
\hline 4 & 31.5 & 27.8 & 22.9 & 17.8 & 1 & 2 & 3 & 1 & 0 & $-17.07^{* * *}$ & .80 & .71 & .921 \\
\hline 5 & 54.5 & 29.9 & 9.5 & 6.1 & 1 & 1 & 2 & 0.5 & 1 & $-15.07^{* * *}$ & .74 & .66 & .923 \\
\hline 6 & 77.9 & 14.4 & 4.3 & 3.3 & 1 & 1 & 1 & 0 & I & $-11.89^{* * *}$ & .73 & .66 & .922 \\
\hline 7 & 45.5 & 28.1 & 14.7 & 11.6 & 1 & 2 & 3 & 1 & 0 & $-17.09^{* * *}$ & .83 & .75 & .918 \\
\hline 8 & 29.1 & 38.0 & 18.1 & 14.7 & 1 & 2 & 3 & 1 & 0 & $-16.44^{* * *}$ & .81 & .73 & .922 \\
\hline 9 & 25.9 & 32.6 & 21.3 & 20.2 & 1 & 2 & 3 & 1 & 0 & $-17.68^{* * *}$ & .86 & .79 & .918 \\
\hline
\end{tabular}

Nota. $\mathrm{N}=807$. Valores de respuesta de los ítems: $1=$ nada, $2=$ algo, $3=$ bastante y 4 = muchísimo.

Cuartiles: primero $\left(\mathrm{Q}^{1}\right)$, segundo o mediana $\left(\mathrm{Q}^{2}\right)$ y tercero $\left(\mathrm{Q}^{3}\right), \mathrm{R}_{\mathrm{SIQ}}=$ rango semiintercuartículo, $\mathrm{A}_{\mathrm{IQ}}=$ asimetría intercuartílica. Discriminabilidad: Estadístico estandarizado de la prueba $\mathrm{U}$ de Mann-Whitney $* * * p<.0001$ (probabilidad de la hipótesis nula de igualdad de rango medio entre los grupos de puntuaciones altas y bajas en la escala). Consistencia interna: $\mathrm{CPS}_{\mathrm{i}, \mathrm{t}}=$ correlación poliserial del ítem con la escala, $\mathrm{CPS}_{\mathrm{i}, \mathrm{t}-\mathrm{i}}=$ correlación poliserial del ítem con el resto de la escala, Ord. $\alpha_{\mathrm{t}-\mathrm{i}}=$ alfa ordinal eliminado el ítem, siendo la Ord. $\alpha_{\mathrm{t}}=.930$. 
Determinación del número de factores

El análisis paralelo de Horn, el de coordenadas óptimas y el del factor de aceleración convergieron en uno tanto en la muestra total como en las de mujeres y hombres, lo que concuerda con la hipótesis de unidimensionalidad. El criterio de Kaiser indicó un factor en la muestra total, pero dos en las muestras de mujeres y hombres. El número de factores fue de dos por los criterios de Velicer en las tres muestras.

\section{Exploración de la estructura de dos factores}

Al extraer dos factores en la muestra total, se explicó el $62.3 \%$ de la varianza total. El primer factor quedó configurado por tres indicadores (ítems 2, 5 y 6) sobre conductas deshonestas (mentir) y con baja aceptación social (ver pornografía), las cuales requerirían una mayor sinceridad para su reconocimiento. Tuvo consistencia interna alta $(\alpha$ ordinal $=.88)$ y validez convergente $(\mathrm{AVE}=.74$ y $\omega=.89)$. El segundo factor quedó conformado por seis indicadores (ítems $1,3,4,7$,

\section{Tabla 4}

Correlación de los ítems de la SSSS_9 con deseabilidad social.

\begin{tabular}{|c|c|c|c|c|c|c|c|c|c|}
\hline \multirow{3}{*}{ SSSS-9 } & \multicolumn{3}{|c|}{ BIDR } & \multicolumn{3}{|c|}{ MI } & \multicolumn{3}{|c|}{$\mathbf{A E}$} \\
\hline & \multirow{2}{*}{$\begin{array}{c}\text { CPS } \\
(\text { CI } 95 \%)\end{array}$} & \multicolumn{2}{|c|}{ Wald } & \multirow{2}{*}{$\begin{array}{c}\text { CPS } \\
(\text { CI } 95 \%)\end{array}$} & \multicolumn{2}{|c|}{ Wald } & \multirow{2}{*}{$\begin{array}{c}\text { CPS } \\
(\text { CI } 95 \%)\end{array}$} & \multicolumn{2}{|c|}{ Wald } \\
\hline & & $\mathbf{W}$ & $p$ & & $\mathbf{W}$ & $p$ & & $\mathbf{W}$ & $p$ \\
\hline \multirow[b]{2}{*}{1} & -.09 & \multirow[b]{2}{*}{5.49} & \multirow[b]{2}{*}{.019} & -.20 & \multirow[b]{2}{*}{28.93} & \multirow[b]{2}{*}{$<.001$} & .07 & \multirow[b]{2}{*}{3.41} & \multirow[b]{2}{*}{.065} \\
\hline & $(-.16,-.01)$ & & & $(-.27,-.13)$ & & & $(-.01, .15)$ & & \\
\hline \multirow[b]{2}{*}{2} & -.28 & \multirow[b]{2}{*}{43.81} & \multirow[b]{2}{*}{$<.001$} & -.34 & \multirow[b]{2}{*}{73.10} & \multirow[b]{2}{*}{$<.001$} & -.12 & \multirow[b]{2}{*}{6.76} & \multirow[b]{2}{*}{.009} \\
\hline & $(-.36,-.20)$ & & & $(-.42,-.26)$ & & & $(-.21,-.03)$ & & \\
\hline \multirow[b]{2}{*}{3} & -.18 & \multirow[b]{2}{*}{19.92} & \multirow[b]{2}{*}{$<.001$} & -.24 & \multirow[b]{2}{*}{35.40} & \multirow[b]{2}{*}{$<.001$} & -.06 & \multirow[b]{2}{*}{1.82} & \multirow[b]{2}{*}{.177} \\
\hline & $(-.26,-.10)$ & & & $(-.32,-.16)$ & & & $(-.14, .03)$ & & \\
\hline \multirow[b]{2}{*}{4} & -.08 & \multirow[b]{2}{*}{4.11} & \multirow[b]{2}{*}{.043} & -.21 & & & .11 & & \\
\hline & $(-.15,-.01)$ & & & $(-.28,-.14)$ & 34.03 & $<.001$ & $(.03, .18)$ & 8.08 & .004 \\
\hline & -.27 & & & -.33 & & & -.11 & & \\
\hline 5 & $(-.34,-.20)$ & 52.46 & $<.001$ & $(-.39,-.26)$ & 86.22 & $<001$ & $(-.19,-.04)$ & 8.40 & .004 \\
\hline & -.32 & & & -.36 & & & -.18 & & \\
\hline 6 & $(-.40,-.24)$ & 64.40 & $<.001$ & $(-.44,-.28)$ & 81.45 & $<.001$ & $(-.26,-.09)$ & 16.00 & $<.001$ \\
\hline & -.17 & & & -.26 & & & -.01 & & \\
\hline 7 & $(-.24,-.09)$ & 19.78 & $<.001$ & $(-.33,-.19)$ & 50.96 & $<.001$ & $(-.09, .07)$ & 0.07 & .798 \\
\hline & -.08 & & & -.20 & & & .09 & & \\
\hline 8 & $(-.15,-.01)$ & 4.91 & .027 & $(-.27,-.13)$ & 32.11 & $<.001$ & $(.02, .17)$ & 5.99 & .014 \\
\hline & -.10 & & & -.22 & & & .07 & & \\
\hline 9 & $(-.18,-.03)$ & 7.06 & .008 & $(-.29,-.15)$ & 36.00 & $<001$ & $(-.01, .15)$ & 3.49 & .062 \\
\hline
\end{tabular}

Nota. $\mathrm{CPS}=$ correlación poliserial, IC $95 \%$ = intervalo de confianza al $95 \%, \mathrm{~W}=$ estadístico $\mathrm{W}$ de Wald, $p=$ probabilidad exacta de $\mathrm{W}$ desde una distribución chi-cuadrado con 1 grado de libertad en un contraste unilateral derecho para la hipótesis nula de $r_{P S}=0$. SSSS-9= escala de búsqueda de sensaciones sexuales, $\mathrm{BIDR}=$ inventario balanceado de deseabilidad social, $\mathrm{MI}=$ manejo de la impresión, y AE = autoengaño. 
8 y 9) sobre la búsqueda de nuevas experiencias y sensaciones sexuales. $\mathrm{Su}$ consistencia interna fue muy alta ( $\alpha$ ordinal $=.91)$ y mostró validez convergente $(\mathrm{AVE}=.64$ y $\omega=.86)$. La varianza compartida entre los dos factores fue muy alta, $55.4 \%$, aunque fue menor que la varianza media extraída de cada uno de ellos, por lo que los dos factores presentaron validez discriminante. El resultado fue muy semejante al extraer dos factores en la muestra de mujeres y hombres, con varianzas compartidas entre los factores de $53.3 \% \mathrm{y}$ $51.8 \%$, respectivamente.

En apoyo de la interpretación dada al primer factor, los ítems 6, 2 y 5 presentaron las correlaciones más altas con manejo de la impresión y la puntuación total del BIDR y fueron los únicos ítems con correlaciones significativas y negativas con autoengaño, lo que concuerda con la afirmación de una mayor sinceridad para reconocer esas conductas deshonestas y con baja aceptación so- cial (Tabla 4). A su vez, el factor conformado por estos tres ítems tuvo correlaciones más altas con deseabilidad social que el otro factor. Sus correlaciones fueron significativas y negativas con una fuerza de asociación moderada con manejo de la impresión, $r_{[807]}=-.32$, IC del $95 \%(-.38,-.25)$, $\mathrm{y}$ baja con la puntuación total del BIDR, $r_{[807]}=$ -.27, IC del $95 \%$ (-.34, -.21), y autoengaño, $r_{\text {[807] }}$ $=-.13$, IC del $95 \%$ (-.20, -.06). El otro factor de seis ítems tuvo correlaciones significativas, negativas y con una fuerza de asociación baja con manejo de la impresión, $r_{[807]}=-.25$, IC del $95 \%$ $(-.31,-.18)$ y la puntuación total del BIDR, $r_{[807]}$ $=-.12$, IC del $95 \%(-.19,-.06)$, pero fue independiente del autoengaño, $r_{[807]}=.06$, IC del $95 \%$ $(-.01, .13)$.

\section{Figura 1}

Modelo de un factor en la muestra total.

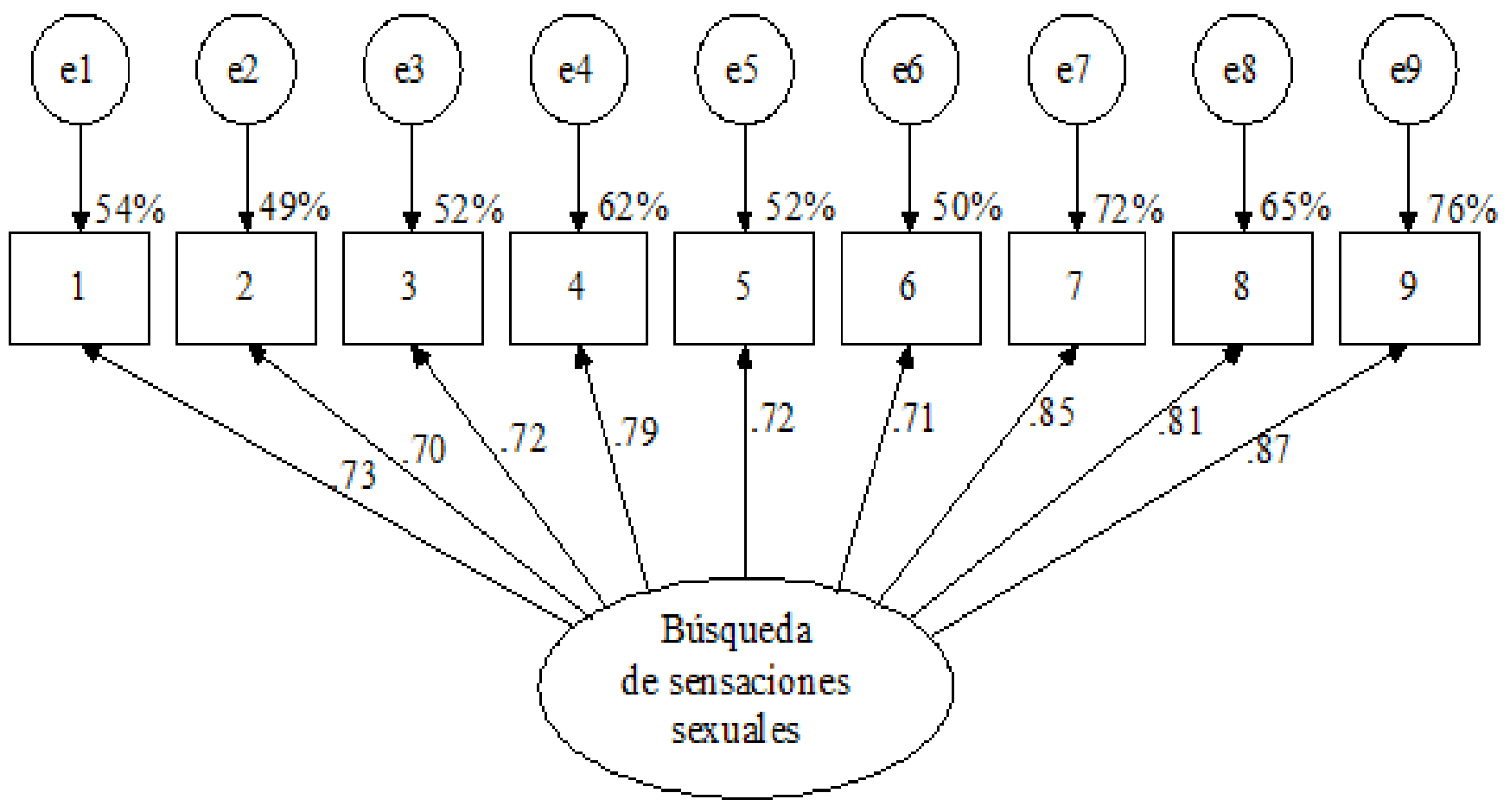


Tabla 5

Índices de ajuste del modelo de un factor y dos factores correlacionados en el contraste unigrupo (muestra total) y multigrupo (sexos).

\begin{tabular}{cccccccc}
\hline \multirow{2}{*}{ Índices } & $\mathbf{1 F}$ & Unigrupo & & \multicolumn{2}{c}{ Multigrupo de 1F } \\
& 2F_AFE & 2F_EXP & SR & CPM & CVE & CRM \\
\hline$\chi^{2}$ & 76.415 & 48.640 & 69.573 & 81.947 & 157.351 & 446.411 & 562.125 \\
\hline$g l$ & 27 & 26 & 26 & 54 & 62 & 63 & 72 \\
\hline$\chi^{2} / g l$ & 2.830 & 1.871 & 2.676 & 1.518 & 2.538 & 7.086 & .807 \\
\hline GFI & .993 & .996 & .994 & .992 & .985 & .957 & .946 \\
\hline AGFI & .989 & .993 & .989 & .987 & .978 & .938 & .932 \\
\hline CFI & .994 & .997 & .995 & .996 & .987 & .948 & .933 \\
\hline NFI & .991 & .994 & .992 & .989 & .979 & .940 & .924 \\
\hline NNFI & .992 & .996 & .993 & .995 & .985 & .940 & .933 \\
\hline RFI & .988 & .992 & .988 & .985 & .975 & .931 & .087 \\
\hline RMSEA & .048 & .033 & .046 & .025 & .044 & .924 \\
\hline SRMR & .075 & .054 & .071 & .088 & .099 & .174 \\
\hline PR & .750 & .722 & .722 & & & .092 \\
\hline
\end{tabular}

Nota. Método: Mínimos cuadrados no ponderados. Datos de entrada: Matriz de correlaciones policóricas. Modelos: $1 \mathrm{~F}=$ un factor con nueve indicadores, $2 \mathrm{~F} \_\mathrm{AFE}=$ dos factores correlacionados desde los resultados del análisis factorial exploratorio: búsqueda de experiencias y sensaciones sexuales (ítems 1,3 , 4, 7, 8 y 9) y conductas censurables (ítems 2, 5 y 6), y 2F_EXP = dos factores correlacionados esperados desde los propuestos para la SSSS-11: búsqueda de sensaciones personales (ítems 1, 8 y 9) y búsqueda de experiencias dependientes de otras personas o estímulos externos (ítems 2,3 , 4, 5, 6 y 7). Contraste de un grupo: en la muestra total de 807 participantes. Contraste multigrupo: separando la muestra de 376 hombres y 431 mujeres y usando modelos anidados: $\mathrm{SR}=$ sin restricciones, $\mathrm{CPM}=$ con restricciones en los pesos de medida, $\mathrm{CVE}=$ con restricciones en la varianzas-covarianzas estructurales, $\mathrm{y}$ CRM $=$ con restricciones en los residuos de medida. Índices de ajuste: $\chi^{2}=$ valor mínimo de la función de discrepancia o chi-cuadrada, $g l=$ grados de libertad de modelo, $\chi^{2} / g l=$ chi-cuadrada relativa, GFI = índice de bondad de ajuste de Jöreskog y Sörbom, AGFI = índice de bondad de ajuste corregido de Jöreskog y Sörbom, CFI = índice comparativo de ajuste de Bentler, NFI = índice de ajuste normado de Bentler y Bonett, NNFI = índice de ajuste no normado de Bentler y Bonett, RFI = índice de ajuste relativo de Bollen, RMSEA = error de aproximación cuadrático medio de Steiger y Lindt, SRMR = error estandarizado cuadrático medio y $\mathrm{PR}=$ razón de parsimonia de James, Mulaik y Brett.

Contraste de los modelos por análisis factorial confirmatorio

Se especificaron tres modelos recursivos con todos sus residuos independientes, uno de un factor con nueve indicadores, y dos de dos factores correlacionados, uno especificado a partir del análisis factorial exploratorio y otro a partir de expectativa conforme a los resultados con la SSSS-11.

En el modelo de un factor, todos los parámetros fueron significativos al ser estimados con un intervalo de confianza bilateral al 95 $\%$. Los pesos de medida fueron mayores que .70 (Figura 1). La varianza media extraída fue de .59 y la confiabilidad compuesta por el coeficiente omega de McDonald fue .93, lo que indica que el factor presentó validez convergente. El ajuste del modelo fue bueno por siete índices y aceptable por dos índices ( $\chi^{2} / g l$ y SRMR), y su parsimonia fue muy alta (Tabla 5).

En el modelo de factores derivado del análisis factorial exploratorio, todos los parámetros fueron significativos. El factor de tres indicadores $(\mathrm{AVE}=.68 \mathrm{y} \omega=.90)$ y el de seis (AVE $=.64 \mathrm{y} \omega=.92)$ mostraron validez convergente, pero carecieron de validez discriminante, al ser la varianza compartida mayor que dos tercios $\left(r^{2}=\right.$ .67) y mayor que la varianza media extraída del factor de seis indicadores. El ajuste del modelo fue bueno por ocho índices y aceptable por uno (SRMR), y su parsimonia fue alta (Tabla 5 y Figura 2).

Igual ocurrió con el modelo especificado a partir de los dos factores correlacionados pro- 
puestos para la SSSS-11 y reducido a los nueve ítems de la SSSS-9: búsqueda de sensaciones personales (ítems 1, 8 y 9) y búsqueda de experiencias dependientes de otras personas o estímulos externos (ítems 2, 3, 4, 5, 6 y 7). Sus parámetros fueron significativos, su ajuste fue bueno por siete índices y adecuado por dos ( $\chi^{2} / g l$ y SRMR) y su parsimonia alta (Tabla 5). Ambos factores tuvieron validez convergente $(\mathrm{AVE}=.685 \mathrm{y} \omega=$ .866 para el factor de tres ítems y AVE $=.644 \mathrm{y}$ $\omega=.906$ para el factor de seis ítems), pero care- cieron totalmente de validez discriminante, al tener una varianza compartida del $94.1 \%$.

Contraste de la invarianza entre ambos sexos.

Al contrastar la invarianza del modelo unifactorial entre ambos sexos, los parámetros fueron significativos en los cuatro modelos anidados en las dos muestras, salvo la varianza del residuo de medida del ítem 6 en el modelo con restric-

\section{Figura 2}

Modelo de dos factores correlacionados, derivado del análisis factorial exploratorio, en la muestra total.

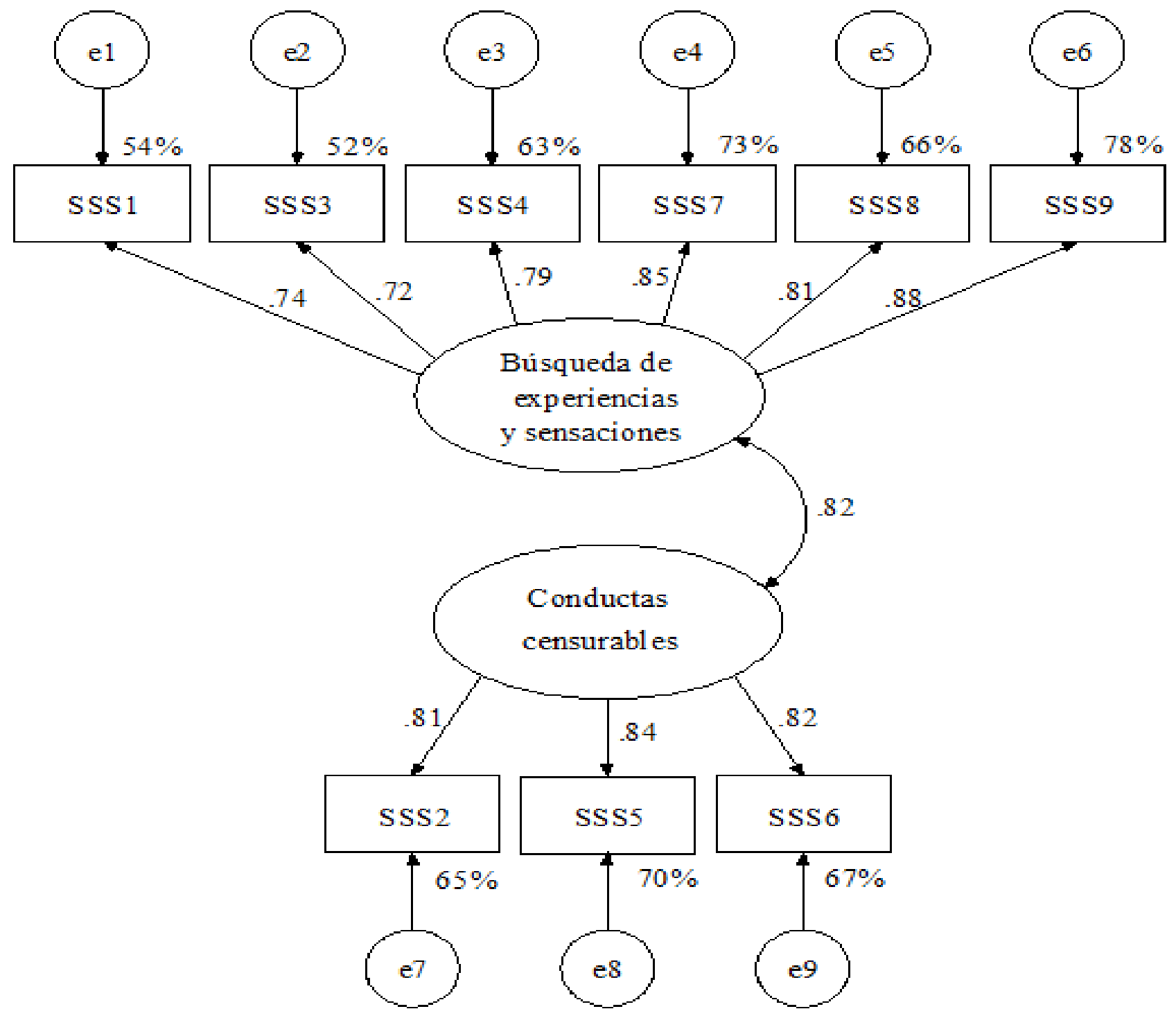


ciones en la varianza estructural en mujeres $\left(\mathrm{S}^{2}{ }_{\mathrm{e} 6}\right.$ $=03$, IC del $95 \%$ : [-.01, .67], $p=.259$ ), al quedar este ítem totalmente explicado por el factor $(\lambda=.95$, IC del $95 \%$ [.85 1.05], $p=.001)$. En el modelo sin restricciones, los parámetros fueron equivalentes entre ambas muestras, salvo las varianzas de los residuos de los ítems 1,2, 3 y 6 , que fueron más altas en hombres; el ajuste fue bueno por todos los índices, salvo por SRMR, en el que fue aceptable. Al restringir los pesos de medida, las mujeres tuvieron valores más altos en los pesos de los ítems 3 y 6 y en la varianza del residuo del ítem 9; los hombres en los pesos de los ítems 4 y 9, la varianza del factor y las varianzas de los residuos de los ítems 2, 3, 5 y 6; empeoró la bondad de ajuste con respecto al modelo anterior $\left(\Delta \chi^{2} / \Delta g l=9.426, \Delta \mathrm{CFI}=.009, \Delta \mathrm{NFI}=.010, \Delta \mathrm{N}-\right.$ $\mathrm{NFI}=.010$ y $\Delta \mathrm{RFI}=.010)$, siendo esta buena por siete índices y aceptable por dos ( $\chi^{2} / g l$ y SRMR). Al restringir adicionalmente la varianza estructural, los pesos de medida de los ítems 2, 3, 5, 6 y 7 fueron más altos en mujeres y las varianzas de los residuos 2, 3, 5, 6, 7 y 8 fueron más altas en hombres; empeoró la bondad de ajuste con respecto al anterior $\left(\Delta \chi^{2} / \Delta g l=289.059, \Delta \mathrm{CFI}=.039, \Delta \mathrm{NFI}\right.$ $=.039, \Delta \mathrm{NNFI}=.045 \mathrm{y} \Delta \mathrm{RFI}=.044)$, siendo esta mala por tres índices ( $\chi^{2} / g l$, SRMR y RMSEA), adecuada por cuatro (NFI, NNFI, CFI y RFI) y buena por dos (GFI y AGFI). Al restringir adicionalmente las varianzas de los residuos de medida, esto es, al hacer los parámetros iguales entre ambas muestras, el ajuste fue malo por dos índices ( $\chi^{2} / g l$ y RMSEA), adecuado por cinco (GFI, NFI, NNFI, CFI y RFI) y bueno por uno (AGFI). La bondad de ajuste empeoró con respecto al modelo anterior $\left(\Delta \chi^{2} / \Delta g l=12.857, \Delta \mathrm{CFI}=.015, \Delta \mathrm{NFI}=\right.$ $.016, \Delta \mathrm{NNFI}=.007$ y $\Delta \mathrm{RFI}=.007$; Tabla 5).

\section{Consistencia interna de la escala}

Al carecer el modelo de dos factores de validez discriminante, se optó por el modelo de un factor, cuyas propiedades de validez convergente y ajuste a los datos fueron buenas y que mostró invarianza en sus pesos de medida entre ambos sexos en el modelo sin restricciones del contraste multigrupo.

La escala integrada por sus nueve ítems presentó una consistencia interna muy alta ( $\alpha$ ordinal $=.93$ en la muestra total, .92 en mujeres y .93 en hombres). No obstante, si se calcula por el coeficiente alfa de Cronbach, la consistencia interna sería alta $(\alpha=.89$ en la muestra total y de hombres y .86 en la de mujeres).

\section{Distribución de la SSSS-9 y diferencia de medias} entre ambos sexos

La distribución de la puntuación total de la SSSS-9 mostró asimetría positiva y no se ajustó a una distribución normal en la muestra total ni en las muestras de mujeres y hombres. La media quedó por encima de la mediana, con mayor concentración de puntuaciones por debajo de la media que por encima, esto es, mayor concentración en puntuaciones bajas (Tabla 6).

La media de los hombres ( $\mathrm{M}=2.05$, IC del $95 \%: 1.97,2.12)$ fue significativamente mayor $\left(t_{[705.11]}=8.21, p<.001\right.$; sin asumir igualdad de varianza con base en la prueba de Levene: $F_{[1,805]}$ $=35.79, p<.001)$ que la de las mujeres $(1.66$, IC del $95 \%$ : 1.61, 1.72) con una diferencia media de 0.39 (IC del $95 \%$ : 0.29, 0.48). El tamaño del efecto del sexo sobre la búsqueda de sensaciones sexuales fue mediano $(g=0.59$, IC del $95 \%$ : $0.45,0.73$ ). 
Tabla 6

Distribución de la SSSS-9 en la muestra total, de mujeres y de hombres.

\begin{tabular}{|c|c|c|c|}
\hline Estadísticos & Mujeres & Hombres & Total \\
\hline $\mathrm{N}$ & 431 & 376 & 807 \\
\hline Mínimo & 1 & 1 & 1 \\
\hline Máximo & 3.89 & 4 & 4 \\
\hline \multirow{2}{*}{ Media } & 1.662 & 2.048 & 1.842 \\
\hline & {$[1.605,1.716]$} & {$[1.972,2.123]$} & {$[1.796,1.892]$} \\
\hline Desviación estándar & 0.575 & 0.737 & 0.683 \\
\hline \multirow{2}{*}{ Asimetría } & 1.009 & 0.491 & 0.804 \\
\hline & {$[0.777,1.224]$} & {$[0.328,0.663]$} & {$[0.672,0.943]$} \\
\hline \multirow{2}{*}{ Curtosis } & 0.751 & -0.559 & 0.011 \\
\hline & {$[0.001,1.534]$} & {$[-0.841,-0.194]$} & {$[-0.327,0.390]$} \\
\hline Percentil 10 & 1 & 1.111 & 1 \\
\hline Percentil 20 & 1.111 & 1.333 & 1.222 \\
\hline Percentil 25 & 1.222 & 1.444 & 1.333 \\
\hline Percentil 30 & 1.333 & 1.556 & 1.333 \\
\hline Percentil 40 & 1.422 & 1.778 & 1.556 \\
\hline Percentil 50 & 1.556 & 2 & 1.667 \\
\hline Percentil 60 & 1.667 & 2.111 & 1.889 \\
\hline Percentil 70 & 1.889 & 2.444 & 2.111 \\
\hline Percentil 75 & 2 & 2.667 & 2.222 \\
\hline Percentil 80 & 2.111 & 2.778 & 2.444 \\
\hline Percentil 90 & 2.444 & 3.111 & 2.889 \\
\hline$\left|D_{\max }\right|$ & $0.137^{* * *}$ & $0.103^{* * *}$ & $0.121^{* * *}$ \\
\hline
\end{tabular}

Nota. Intervalos de confianza del $95 \%$, creados por el procedimiento de remuestreo con la extracción de 1000 muestras aleatorias. $\left|\mathrm{D}_{\max }\right|=$ Estadístico de contraste de la prueba de Kolmogorov-Smirnov. La probabilidad fue calculada con las tablas de Lilliefors. *** $p<.001$ (a una cola).

Validez de constructo concurrente y efecto de la deseabilidad social

En la muestra conjunta, la correlación de la SSSS-9 fue significativa, positiva y alta con la puntuación total de la escala de conducta infiel y sus factores de infidelidad sexual, deseo de infidelidad emocional y deseo de infidelidad sexual. Fue moderada con el factor de infidelidad emo- cional y la escala de actitud hacia la exclusividad marital. Estas seis correlaciones fueron estadísticamente equivalentes entre ambos sexos por la prueba de Fisher (Tabla 7).

En la muestra conjunta, la correlación de la SSSS-9 fue significativa, negativa y baja con la puntuación total de la escala de deseabilidad social y su factor de manejo de la impresión, y fue independiente del factor de autoengaño. Estas 
Tabla 7

Correlaciones de la SSSS-9 con las escalas de actitud hacia la exclusividad marital, conducta infiel y deseabilidad social.

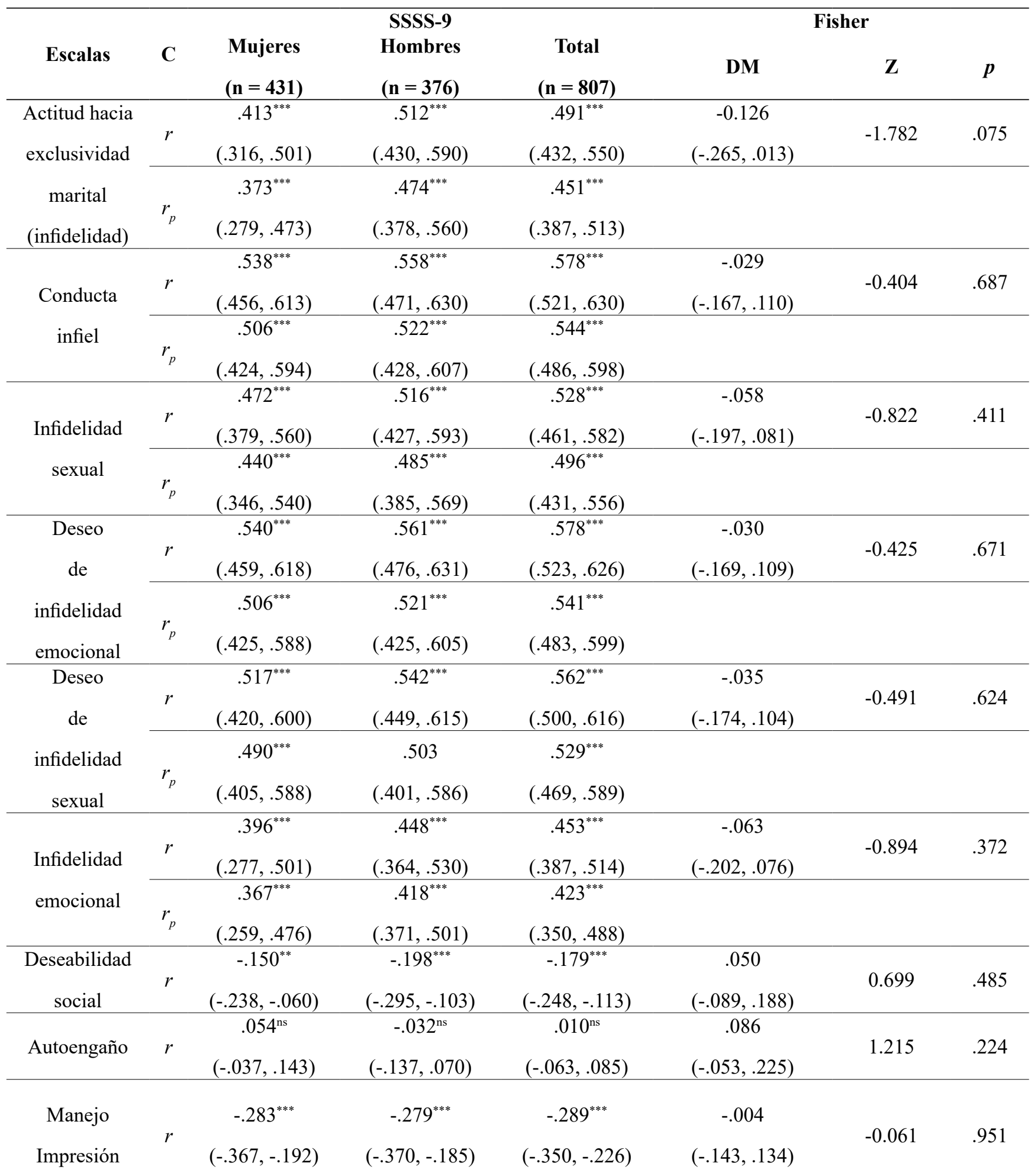

Nota. $\mathrm{C}=$ coeficiente de correlación: $r=$ coeficiente de correlación producto-momento de Pearson y $r_{p}=$ coeficiente de correlación parcial de Fisher, parcializando el manejo de la impresión. DM $=$ diferencia media. Intervalos de confianza estimados con la extracción de 1,000 muestras aleatorias. Significación en un contraste bilateral por la prueba $\mathrm{Z}$ de Fisher: $\mathrm{ns}=p>.05$, * $p<.05$, ** $p<.01$, *** $p<.001$. 
tres correlaciones fueron estadísticamente equivalentes entre ambos sexos (Tabla 7).

Al parcializar el manejo de la impresión, las correlaciones de SSSS-9 con la escala de conducta infiel y sus cuatro factores y la escala de actitud hacia la exclusividad marital siguieron siendo significativas con fuerzas de asociación de altas a moderadas, disminuyendo en cuatro o menos centésimas (Tabla 7).

\section{Discusión}

Como primer objetivo se planteó comprobar la consistencia interna, discriminabilidad y variabilidad de los ítems. Como en estudios previos (De Oliveira-Santos et al., 2017; Kalichman et al., 1994; Teva \& Paz-Bermúdez, 2008), los ítems mostraron consistencia interna. Asimismo, tuvieron capacidad para diferenciar al grupo de puntuaciones altas y bajas en la escala con un poder discriminativo grande en siete de los nueve ítems y mediano en dos, lo que anteriormente no se había comprobado. Además, ninguno presentó efecto suelo ( $80 \%$ o más de los casos en el primer valor) o techo ( $80 \%$ o más de los casos en el último valor) y la variabilidad fue alta en la mayoría de los ítems. Conforme con la expectativa (Gadermann et al., 2014), los indicadores de consistencia interna fueron más altos que en estudios previos por el uso de un método más adecuado a la naturaleza ordinal de los ítems. A su vez, esta mejora en los índices se puede atribuir a la unidimensionalidad que presenta la versión original de la escala frente al modelo de dos factores de la versión revisada (Ballester-Arnal et al., 2018; De Oliveira-Santos et al., 2017; Santos-Iglesias et al., 2018). Los ítems 2 y 3, eliminados en la revisión de la escala (Kalichman \& Rompa, 1995), no resultaron débiles en su consistencia interna. Su nivel fue semejante a los ítems 1,5 y 6 , siendo los más fuertes en esta propiedad los cuatro ítems restantes, los ítems 4, 7, 8 y 9.

Se enunció como segundo objetivo determinar el número de factores. Conforme con la expectativa de Kalichman et al. (1994), los tres criterios empíricos más válidos en los estudios de simulación convergieron en un factor, el cual quedó definido por pesos de medida mayores que .70, lo que proporciona garantías de que es el verdadero número de factores (Courtney, 2013).

No obstante, el criterio de Velicer, considerado también válido, junto con el criterio de Kaiser, considerado el menos válido, convergieron en dos, conforme al número de factores que presenta la versión revisada de la escala (Ballester-Arnal et al., 2018; De Oliveira-Santos et al., 2017; Santos-Iglesias et al., 2018). Al explorar el modelo bidimensional, se configuraron dos factores consistentes y con validez convergente, aunque con una varianza compartida muy alta. Estos dos factores difieren en contenido de los obtenidos con la SSSS-11. No separan a los ítems sobre sensaciones con más acento en lo personal de los ítems sobre experiencias dependientes de otras personas o estímulos externos, sino que separan a los ítems con más sesgo de deseabilidad social por su contenido (mentir y ver pornografía) de los ítems con menos potencial de rechazo social, como muestra la relación de los ítems con el BIDR y sus factores de manejo de la impresión y autoengaño.

El tercer objetivo propuesto fue contrastar los modelos y el cuarto comprobar la invarianza de los mismos entre mujeres y hombres. El ajuste a los datos del modelo unidimensional fue bueno por mínimos cuadrados no ponderados, el cual es un método más adecuado para variables ordinales que el de máxima verosimilitud (Byrne, 2016), como se emplea en otros estudios (Ballester-Arnal et al., 2018; De Oliveira-Santos et al., 2017; Santos-Iglesias et al., 2018). El modelo de un factor fue válido para mujeres y hombres por 
la significación de las estimaciones, el tamaño del efecto muy grande del factor sobre los ítems y el buen ajuste del modelo sin restricciones y con restricciones en los pesos de medida (Byrne, 2016). No obstante, el modelo no fue estrictamente invariante. Para poder hablar de invarianza, la estimación por intervalo (al $95 \%$ ) de cada parámetro (peso de medida, error de medida y varianza del factor) debería solaparse en la muestra de mujeres y hombres en los cuatro modelos anidados en restricciones, las diferencias entre los valores mínimos de la función de discrepancia entre los cuatro modelos anidados deberían ser estadísticamente equivalentes con un nivel de significación unilateral de .05 , el cociente entre la diferencia de los valores mínimos de la función de discrepancia y la diferencia de sus grados de libertad $\left(\Delta \chi^{2}\right)$ $\Delta g l$ ) debería ser menor que 2, y las diferencias entre los índices de ajuste relativos ( $\triangle \mathrm{GFI}, \triangle \mathrm{AG}$ FI, $\Delta$ NFI y $\Delta$ RFI) menores que .01 (Byrne, 2016).

También se contrastó el modelo de dos factores revelado por el análisis factorial exploratorio. Aunque su ajuste e invarianza entre ambos sexos fue mejor que el modelo de un factor, el análisis factorial confirmatorio evidenció claramente el problema de validez discriminante, o exceso de varianza compartida entre los factores, que ya parecía entreverse en el análisis factorial exploratorio. Un modelo más complejo, como el modelo de dos factores correlacionados, en comparación con un modelo más simple y parsimonioso como es el de un factor, suele mostrar mejores índices de ajuste, por lo que es muy importante comprobar la validez discriminante entre los factores (Byrne, 2016; Kóbor, Takács, \& Urbán, 2013), lo que fa1ló con los nueve ítems de la SSSS-9, de ahí que se descartó.

En el caso del modelo de dos factores esperado desde las propuestas para la SSSS-11 (Ba1lester-Arnal et al., 2018; De Oliveira-Santos et al., 2017; Santos-Iglesias et al., 2018), la falta de validez discriminante fue mucho más fuerte, aparte de no ser revelado por el análisis factorial exploratorio, lo que indica que es totalmente forzado para los nueve ítems de la SSSS-9, de ahí que también se descartó.

Se formuló como quinto objetivo comprobar la consistencia interna de la SSSS-9. En la presente muestra de adultos mexicanos casados o cohabitantes con parejas del sexo contrario, la consistencia interna de los nueve ítems de la SSSS-9 fue muy alta, al ser calculada por el coeficiente alfa ordinal, tanto en la muestra conjunta como al separar mujeres y hombres. Una consistencia interna muy alta apunta hacia un único factor subyacente (Sijtsma, 2015), como revelaron el análisis paralelo de Horn, coordenadas óptimas y factor de aceleración, por lo que este resultado apoya a la conclusión de unidimensionalidad. No obstante, con esta misma versión en una muestra de 106 hombres con orientación homosexual, Kalichman et al. (1994) obtuvieron una consistencia interna aceptable. Cabe señalar que la consistencia interna de la SSSS, calculada por el alfa de Cronbach y con independencia de la versión usada, es menor en las muestras de hombres que tienen sexo con hombres (Kalichman et al., 1994; primer estudio de Kalichman \& Rompa, 1995; Valdez et al., 2016), lo que resulta en valores aceptables; por el contrario, es mayor en población general (segundo estudio de Kalichman \& Rompa, 1995) y estudiantes universitarios (De Oliveira-Santos et al., 2017; Santos-Iglesias et al., 2018). En estas últimas poblaciones se alcanzan valores altos, como en este estudio con personas heterosexuales casadas o que cohabitan.

Se enunció como sexto objetivo describir la distribución de la SSSS-9. La distribución esperada para rasgos de personalidad es la distribución normal (Lyon, 2012). Este no fue el caso de la SSSS-9. Su distribución se desvió de la normalidad debido a la presencia de asimetría positiva. 
Las colas de la distribución fueron asimétricas y la cola larga apareció hacia la derecha, lo que provocó que haya más casos por debajo de la media aritmética que por encima. Esto indica que hay un grupo pequeño de personas con un rasgo de búsqueda de sensaciones sexuales muy definido o alejado del promedio que cae en lo que podría calificarse como conducta sexual compulsiva (Kalichman et al., 1994). Si se observa el perfil del histograma, este no fue acampanado, sino que tuvo forma de escalera descendiente, aproximándose más al perfil de las escalas que evalúan rasgos psicopatológicos, como las escalas clínicas del Inventario Multifásico de Personalidad de Minnesota (Ben-Porath \& Tellegen, 2016). Por tanto, es mejor realizar la baremación de la escala a través de medidas de posición o percentil en lugar de usar puntuaciones $\mathrm{T}$ basadas en la media aritmética y desviación estándar (Ben-Porath \& Tellegen, 2016). Dentro de esta población de adultos mexicanos casados o cohabitantes con parejas del sexo contrario que residen en Monterrey, una puntuación mayor o igual de 2.11 en mujeres y 2.78 en hombres (percentil 80) indica que el rasgo de la búsqueda de sensaciones sexuales está muy definido o destacado.

Un rasgo de búsqueda de sensaciones más definido en hombres que en mujeres es un hecho bien establecido (Cross et al., 2013), lo que aplica al constructo de la búsqueda de sensaciones sexuales (Kalichman \& Rompa, 1995) y se acentúa por la mayor libertad sexual que goza el hombre en la mayoría de las culturas, incluida la occidental (Ballester-Arnal et al., 2018; De Oliveira-Santos et al., 2017; Kalichman \& Rompa, 1995; Santos-Iglesias et al., 2018). Los presentes datos con la versión de nueve ítems confirmaron esta expectativa. Además, mostraron que el tamaño del efecto del sexo sobre la búsqueda de sensaciones sexuales es mediano, como también se ha observado en las versiones de 10 ítems (Gaither
\& Sellbom, 2003) y de 11 ítems (Ballester-Arnal et al., 2018; De Oliveira-Santos et al., 2017; Kalichman \& Rompa, 1995; Santos-Iglesias et al., 2018; Teva \& Paz-Bermúdez, 2008).

Se propuso como séptimo objetivo comprobar la validez de constructo concurrente en relación con la conducta infiel y actitud hacia la exclusividad marital. Se confirmaron las expectativas de validez de constructo concurrente (Kogan et al., 2015). Una mayor búsqueda de sensaciones actuó como un factor de riesgo con un tamaño del efecto grande sobre desear y tener parejas sexuales concurrentes y desear emocionalmente otras parejas. Su tamaño del efecto fue moderado con conductas de implicación emocional con otras parejas y actitud favorable hacia la concurrencia de parejas. Aunque estas correlaciones aparentemente fueron más altas en hombres que en mujeres, al contrastar la diferencia con la prueba $\mathrm{Z}$ de Fisher para datos emparejados de dos muestras independientes y calcular el intervalo de confianza de la diferencia entre las correlaciones por un procedimiento paramétrico de remuestreo, las diferencias resultaron nulas, es decir, no significativas. La búsqueda de sensaciones sexuales es un rasgo más definido en hombres, pero su fuerza de asociación con la actitud hacia la exclusividad marital y conducta infiel es equivalente en hombres y mujeres, como previamente habían reportado Gaither y Sellbom (2003) con permisividad sexual.

El octavo objetivo fue observar el efecto de la deseabilidad social sobre la SSSS-9. Esta variable de sesgo tuvo un efecto pequeño sobre la búsqueda de sensaciones sexuales debido a su factor de manejo de la impresión, sin resultar este sesgo diferencial entre ambos sexos. La varianza compartida entre el manejo de la impresión y la búsqueda de sensaciones sexuales fue menor del $9 \%$. Al parcializar el efecto del manejo de la impresión en las correlaciones para establecer la 
validez de constructo concurrente, estas siguieron siendo significativas y descendieron en un máximo de cuatro centésimas, lo que indica la sustantividad de las mismas y fortalece la prueba de validez de constructo concurrente.

Una primera limitación del presente estudio es que las inferencias sólo son aplicables a adultos mexicanos casados o cohabitantes con una pareja del sexo contrario que residen en Monterrey. Con la debida precaución, se podrían generalizar o usar como hipótesis para poblaciones afines, esto es, urbanas de cultura latina. Una segunda limitación es que los datos no permiten inferencias causales, ni estimar la confiabilidad temporal de las puntuaciones, ni la estabilidad temporal de la estructura factorial, ya que se usó un diseño ex post facto transversal. Una tercera limitación es la naturaleza de autorreporte de los datos, por lo que estos pueden diferir de los obtenidos con técnicas proyectivas, observacionales o procedimientos para evaluar actitudes implícitas.

Se concluye que, en adultos mexicanos casados o cohabitantes con una pareja del sexo contrario residentes en Monterrey, la escala SSSS-9 presenta una consistencia interna global muy alta por el coeficiente alfa ordinal y alta por el coeficiente alfa de Cronbach. Sus nueve ítems cumplen con las propiedades de consistencia interna y discriminabilidad y no muestran anomalías distribucionales del tipo efecto techo, efecto suelo o bimodalidad en valores extremos. La estructura de la escala es unidimensional como indican la convergencia del análisis paralelo de Horn y coordenadas óptimas en un factor, el tamaño del efecto muy grande del factor sobre sus nueve indicadores, la validez convergente y consistencia interna del factor único y el buen ajuste a los datos del modelo unifactorial. El modelo de un factor es válido para ambos sexos, aunque no estrictamente invariante. La distribución de la escala presenta sesgo hacia la cola derecha, con más valores por debajo que por encima de la media aritmética, por lo que no se ajusta a una distribución normal y por ende su estandarización puede realizarse a través de puntuaciones de percentil. La media de los hombres es mayor que la de las mujeres, el tamaño del efecto del sexo sobre la escala es mediano, por lo que se requieren baremos diferenciales para mujeres y hombres. La SSSS-9 presenta validez de constructo concurrente en relación con conducta infiel y actitud hacia la exclusividad marital. La deseabilidad social impacta sobre la SSSS-9 con un tamaño del efecto pequeño y debido a su factor de manejo de la impresión.

Se sugiere el uso de la SSSS-9 como una medición unidimensional de la búsqueda de sensaciones sexuales en el estudio de las parejas casadas o cohabitantes, ya que constituye un factor de riesgo de infidelidad con un tamaño del efecto grande. Se sugiere estudiar la estabilidad de sus puntuaciones y de la estructura unifactorial. En futuros estudios con la SSSS-9 sería interesante comprobar si el modelo de dos factores (búsqueda de sensaciones y experiencias sexuales y conductas censurables) posee validez discriminante en otras poblaciones.

\section{Referencias}

American Psychological Association (2017). Ethical principles of psychologists and code of conduct. With the 2016 amendment to standard 3.04. Washington, DC: American Psychological Association.

Ballester-Arnal, R., Ruiz-Palomino, E., Espada-Sánchez, J. P., Morell-Mengual, V., \& Gil-Llario, M. D. (2018). Psychometric properties and validation of the Sexual Sensation Seeking Scale in Spanish adolescents: Brief screening method for use in research and clinical practice. Personality and Individual Differences, 122, 47-54. doi: 10.1016/j.paid.2017.10.006

Ben-Porath, Y. S., \& Tellegen, A. (2016). Part 3: restandar- 
dizing the MMPI. Minneapolis, MN: University of Minnesota Press.

Byrne, B. M. (2016). Structural equation modeling with AMOS: basic concepts, applications, and programming (3a ed.). New York, NY: Routledge.

Courtney, M. G. R. (2013). Determining the number of factors to retain in EFA: Using the SPSS R-menu v2.0 to make more judicious estimations. Practical Assessment, 18, 1-14 (artículo 8). doi: 10.2147/JHL. S35483

Cross, C. P., Cyrenne, D. L. M., \& Brown, G. R. (2013). Sex differences in sensation-seeking: A meta-analysis. Scientific Reports, 3 (artículo 2486). doi: 10.1038/ srep02486

De Oliveira-Santos, M. J., Soares-Ferreira, E. M., Carvalho-Duarte, J. C., \& Ferreira, M. M. (2017). Adaptação portuguesa e validação da Sexual Sensation Seeking Scale para estudantes do ensino superior. Revista de Enfermagem Referência, 4(15), 11-20. doi: 10.12707/riv17054

Gadermann, A., Guhn, M., \& Zumbo, B. D. (2014). Ordinal alpha. En A. C. Michalos (Ed.), Encyclopedia of quality of life and well-being research (pp. 4513-4515). Dordrecht: Springer Netherlands. doi: 10.1007/97894-007-0753-5 2025

Gaither, G. A., \& Sellbom, M. (2003). The Sexual Sensation Seeking Scale: Reliability and validity within a heterosexual college student sample. Journal of Personality Assessment, 81(2), 157-167. doi: 10.1207/ s15327752jpa8102_07

Grubbs, J. B., Exline, J. J., Pargament, K. I., Hook, J. N., $\&$ Carlisle, R. D. (2015). Transgression as addiction: Religiosity and moral disapproval as predictors of perceived addiction to pornography. Archives of Sexual Behavior, 44(1), 125-136. doi: 10.1007/s10508013-0257-z

Huang, C. E., Cassels, S. L., \& Winer, R. L. (2015). Self-reported sex partner dates for use in measuring concurrent sexual partnerships: Correspondence between two assessment methods. Archives of Sexual Behavior, 44(4), 873-883. doi: 10.1007/s10508-014-
0414-Z

Iglesias, M. C., \& Reyes, G. (2017). 30 años del VIH/SIDA. Perspectiva desde México. Ciudad de México: Centro de Investigación en Enfermedades Infecciosas y Fundación México Vivo.

Instituto Nacional de Estadística, Geografía e Informática (2016). Panorama sociodemográfico de Nuevo León 2015. Ciudad de México: INEGI. Recuperado de http://www.beta.inegi.org.mx

Kalichman, S. C., Adair, V., Rompa, D., Multhauf, K., Johnson, J., \& Kelly, J. (1994). Sexual Sensation-Seeking Scale: Development and predicting AIDS-risk behavior among homosexually active men. Journal of Personality Assessment, 62(3), 385-397. doi: 10.1207/s15327752jpa6203_1

Kalichman, S. C., \& Rompa, D. (1995). Sexual Sensation Seeking and Sexual Compulsivity Scales: Reliability, validity, and predicting HIV risk behaviors. Journal of Personality Assessment, 65(3), 586-601. doi: 10.1207/s15327752jpa6503_16

Kóbor, A., Takács, A., \& Urbán, R. (2013). The bifactor model of the Strengths and Difficulties Questionnaire. European Journal of Psychological Assessment, 29(4), 299-307. doi: 10.1027/1015-5759/a000160

Kogan, S. M., Cho, J., Barnum, S. C., \& Brown, G. L. (2015). Correlates of concurrent sexual partnerships among young, rural African American men. Public Health Reports, 130(4), 392-399. doi: 10.1177/003335491513000418

Lyon, A. (2012). Mathematical explanations of empirical facts, and mathematical realism. Australasian Journal of Philosophy, 90(3), 559-578. doi: 10.1080/00048402.2011.596216

Moral, J., García, C. H., \& Antona, C. J. (2012). Traducción y validación del Inventario Balanceado de Deseabilidad Social al Responder en una muestra probabilística de estudiantes universitarios mexicanos. Revista de Psicología GEPU, 3(2), 54-72. Recuperado de https://revistadepsicologiagepu.es.tl

Muise, A., Stanton, S. C. E., Kim, J. J., \& Impett, E. A. (2016). Not in the mood? Men under- (not over-) 
perceive their partner's sexual desire in established intimate relationships. Journal of Personality and Social Psychology, 110(5), 725-742. doi: 10.1037/ pspi0000046

Romero-Palencia, A., Rivera-Aragón, S., \& Díaz-Loving, R. (2007). Desarrollo del Inventario Multidimensional de Infidelidad (IMIN). Revista Iberoamericana de Diagnóstico y Evaluación Psicológica, 23(1), 121-147. Recuperado de http://www.aidep.org/pt/ pdf/4596/459645446008.pdf

Santos-Iglesias, P., Moyano, N., Castro, A., Granados, M. R., \& Sierra, J. C. (2018). Validation of the Sexual Sensation Seeking Scale in Spanish samples. Journal of Personality Assessment, 100(4), 421-430. doi: 10.1080/00223891.2017.1328424

Selterman, D., \& Koleva, S. (2015). Moral judgment of close relationship behaviors. Journal of Social and Personal Relationships, 32(7), 922-945. doi: 10.1177/0265407514554513

Sijtsma, K. (2015). Delimiting coefficient $\alpha$ from internal consistency and unidimensionality. Educational Measurement: Issues and Practice, 34(4), 10-13. doi: 10.1111/emip.12099

Streiner, D. L., Norman, G. R., \& Cairney, J. (2015). Health measurement scales: A practical guide to their development and use (5a ed.). New York, NY: Oxford University.

Téllez, A., García, C. H., \& Corral-Verdugo, V. (2015). Effect size, confidence intervals and statistical power in psychological research. Psychology in Russia: State of the Art, 8(3), 27-46. doi: 10.11621/ pir.2015.0303

Teva, I., \& Paz-Bermúdez, M. (2008). Adaptación castellana y propiedades psicométricas de la Escala de Búsqueda de Sensaciones Sexuales en adolescentes españoles. Revista Mexicana de Psicología, 25(1), 129-137. Recuperado de http://www.redalyc.org/articulo.oa?id $=243016300010$

Valdez, C., Moral, J., Onofre, D. J., Castillo, L., Benavides, R., \& López, F. (2016). Búsqueda de sensaciones sexuales en hombres que tienen sexo con hombres.
Medicina Universitaria, 18(Supl. 1), 87. Recuperado de http://www.elsevier.es/en-revista-medicina-universitaria-304-seccion-xxviii-congreso-nacional-investigacion-medicina-66641

Weis, D. L., \& Felton, J. R. (1987). Marital exclusivity and the potencial for future marital conflict. Social Work, 32(1), 45-49. doi: 10.1093/sw/32.1.45

Xu, W., Zheng, L., Liu., Y., \& Zheng, Y. (2016). Sexual sensation seeking, sexual compulsivity, and high-risk sexual behaviours among gay/bisexual men in Southwest China. AIDS Care, 28(9), 1138-1144. doi: 10.1080/09540121.2016.1153587 
Anexo

Escala de búsqueda de sensaciones sexuales (SSSS-9).

Señale qué tanto le caracterizan las siguientes afirmaciones en la siguiente escala.

\begin{tabular}{|c|c|c|c|c|}
\hline $\begin{array}{c}3 \\
\text { Bastante } \\
\end{array}$ & & & & $\begin{array}{c}4 \\
\text { Muchísimo }\end{array}$ \\
\hline 1. Me gustan los encuentros sexuales desinhibidos y salvajes & 1 & 2 & 3 & 4 \\
\hline $\begin{array}{l}\text { 2. He hecho promesas sin la intención de mantenerlas para que otra persona tenga } \\
\text { sexo conmigo }\end{array}$ & 1 & 2 & 3 & 4 \\
\hline 3. Siento curiosidad en tener sexo anal sin condón & 1 & 2 & 3 & 4 \\
\hline 4. Disfruto de la compañía de una persona sensual & 1 & 2 & 3 & 4 \\
\hline 5. Disfruto ver películas pornográficas & 1 & 2 & 3 & 4 \\
\hline $\begin{array}{l}\text { 6. He dicho cosas que no son exactamente ciertas para lograr que una persona tenga } \\
\text { sexo conmigo }\end{array}$ & 1 & 2 & 3 & 4 \\
\hline 7. Estoy interesada/o en probar nuevas experiencias sexuales & 1 & 2 & 3 & 4 \\
\hline 8. Me gusta explorar mi sexualidad & 1 & 2 & 3 & 4 \\
\hline 9. Me gustan las experiencias y sensaciones sexuales nuevas y excitantes & 1 & 2 & 3 & 4 \\
\hline
\end{tabular}

